\title{
Improvement in social and cognitive functioning associated with paliperidone extended-release treatment in patients with schizophrenia: a 24-week, single arm, open-label study
}

\author{
This article was published in the following Dove Press journal: \\ Neuropsychiatric Disease and Treatment \\ 24 August 2016 \\ Number of times this article has been viewed
}

\author{
Chuan Shi, ${ }^{1-4}$ Shu Qiao Yao, ${ }^{5}$ \\ Yi Feng $X u,{ }^{6}$ Jian Guo Shi, ${ }^{7}$ \\ Xiu Feng $\mathrm{Xu},{ }^{8}$ Cong Pei \\ Zhang, ${ }^{9}$ Hua Jin, ${ }^{10}$ Xin $\mathrm{Yu}^{1-4}$ \\ 'Clinical Research Center, Peking \\ University Sixth Hospital, ${ }^{2}$ Clinical \\ Research Center, Peking University \\ Institute of Mental Health, ${ }^{3} \mathrm{Key}$ \\ Laboratory of Mental Health, Ministry \\ of Health (Peking University), \\ ${ }^{4}$ National Clinical Research Center \\ for Mental Disorders (Peking \\ University Sixth Hospital), Beijing, \\ ${ }^{5}$ Clinical Center of Psychology, The \\ Second Xiangya Hospital, Central \\ South University, Changsha, Hunan \\ Province, ${ }^{6}$ Department of Psychiatry, \\ Institute of Mental Health, Shanghai \\ Jiao Tong University, Shanghai, \\ ${ }^{7}$ Department of Psychiatry, Xi'an \\ Mental Health Center, Xian, Shanxi \\ Province, ${ }^{8}$ Department of Psychiatry, \\ The First Affiliated Hospital of \\ Kunming Medical School, Kunming, \\ Yunnan Province, ${ }^{9}$ Department \\ of Psychiatry, The First Haerbin \\ Psychiatric Hospital, Haerbin, \\ Heilongjiang Province, People's \\ Republic of China; ${ }^{10}$ Department of \\ Psychiatry, University of California \\ at San Diego, La Jolla, San Diego, \\ CA, USA
}

Correspondence: Xin Yu Clinical Research Center, Peking University Sixth Hospital, 5I Hua Yuan Bei Road, Haidian District, Beijing, I00191, People's Republic of China Tel +86 I0 I39 I0229255 Fax +86 10 62384009 Email yuxin@bjmu.edu.cn
Purpose: This single-arm, open-label study aimed to explore the effects of extended-release paliperidone on social and cognitive function in patients with schizophrenia.

Methods: Paliperidone extended-release (flexible dose ranging from 3 to $12 \mathrm{mg}$ /day orally) was administered for 24 weeks in patients with schizophrenia. Patient function was assessed using the personal and social performance scale, measurement and treatment research to improve cognition in schizophrenia initiative-consensus cognitive battery, positive and negative syndrome scale, and clinical global impression-severity.

Results: Ninety patients were included in the full analysis set, while 72 patients were included in the per protocol set. The personal and social performance score was $54.3 \pm 14.3$ at baseline, and significantly increased to $73.4 \pm 12.6$ at week $24(P<0.001)$. For the measurement and treatment research to improve cognition in schizophrenia initiative-consensus cognitive battery assessment, six of the nine individual subtests, six of the seven cognitive domains, and total cognitive scores improved significantly $(P<0.05)$ between baseline and endpoint. positive and negative syndrome scale total scores and clinical global impression-severity scores decreased gradually $(P<0.001)$ from week 4 to the conclusion of the study.

Conclusion: Paliperidone extended-release treatment significantly improves social and neurocognitive function as well as symptoms in Chinese patients with schizophrenia.

Keywords: paliperidone, schizophrenia, neurocognition, social function

\section{Introduction}

Paliperidone is an atypical antipsychotic utilized for the treatment of schizophrenia and schizoaffective disorders, acting mainly as a monoaminergic antagonist at dopamine-2 $\left(\mathrm{D}_{2}\right)$ and serotonin $2 \mathrm{~A}\left(5 \mathrm{HT}_{2 \mathrm{~A}}\right)$ receptors. The recently developed paliperidone extended-release (ER) tablet utilizes the osmotic release oral system technology, which allows for precise yet sustained release of the drug over a 24-hour period. The once daily dosing of this type of drug is often more convenient. ${ }^{1}$

Previous studies have demonstrated that paliperidone ER can significantly improve social functioning, health-related quality of life, and disease severity in patients undergoing treatment for acute schizophrenia..$^{2-4}$ Second-generation antipsychotics such as paliperidone improve social functioning by reducing psychotic symptoms with fewer adverse interactions than previously developed medications. Furthermore, a systematic review of eleven comparative studies revealed that paliperidone ER resulted in significantly greater improvements in functionality as assessed by the Personal 
and Social Performance Scale (PSP) than those achieved with comparative antipsychotics, including risperidone, olanzapine, and aripiprazole. ${ }^{5}$

Though the effects of paliperidone ER on social and personal functioning have been somewhat well-documented, similar effects with respect to improvements in cognitive function remain unverified. However, in a systematic review of 12 studies, Houthoofd et al documented the effects of risperidone on neurocognitive function in patients with schizophrenia and schizoaffective disorder. ${ }^{6}$ These patients exhibited significant improvements with regard to the cognitive domains of processing speed, attention/vigilance, verbal and visual learning and memory, and reasoning and problem-solving after 12-54 weeks of treatment with risperidone. However, the effect of risperidone on social cognition in patients with schizophrenia remains controversial due to conflicting results. For example, Sergi et al failed to observe any correlation between risperidone treatment and improvements in social cognition, in contrast to results obtained by Harvey et al and Maat et al. ${ }^{7-9}$ Furthermore, some newer antipsychotics were not investigated in these systematic reviews, including paliperidone ER.

Paliperidone is the primary active metabolite of risperidone, and both drugs act via similar pathways. Paliperidone, however, has a stronger antagonistic effect than risperidone on adrenergic $\left(\alpha_{2}\right)$, dopamine-3 $\left(\mathrm{D}_{3}\right)$, and serotonin $7\left(5-\mathrm{HT}_{7}\right)$ receptors, which may increase concentrations of norepinephrine, serotonin (5-HT), and acetylcholine at the synaptic cleft. These neuropharmacological characteristics suggest that paliperidone may be more effective than risperidone for improving neurocognitive function. Indeed, one study revealed that, compared with risperidone, paliperidone exhibits certain advantages in terms of improving verbal memory. ${ }^{10}$ However, the effects of paliperidone ER treatment on overall neurocognitive function in Chinese patients with schizophrenia remain to be fully elucidated.

The present study aimed to explore improvements in neurocognitive and social function associated with paliperidone ER treatment, as well as the relationship of these improvements to functional outcomes in Chinese patients with schizophrenia.

\section{Participants and methods Study design}

We conducted a 24-week, open, single-arm, multicenter prospective study including 95 patients with schizophrenia. All participants were recruited from one of six different sites throughout China: IMHPU; the Second Xiangya Hospital,
Central South University; Institute of Mental Health, Shanghai Jiao Tong University; Xian Mental Health Center; the First Haerbin Psychiatric Hospital; and the First Affiliated Hospital of Kunming Medical University. The recruitment procedure was similar for all six sites. Participants were contacted by the recruiter of each site, and potential participants were identified by colleagues and friends. No extra incentives were provided for participation in the study. All participants provided written informed consent. The present study was approved by the Ethics Committee of Peking University Sixth Hospital.

The complete study consisted of a screening period, a 24-week study period, and an optional extension phase. This manuscript reports results from the 24-week study period.

\section{Patients}

Criteria for inclusion were as follows: 1 ) age $20-45 ; 2$ ) $\geq 5$ years of education; 3) ability to read and understand Chinese; 4) diagnosed with schizophrenia according to Diagnostic and Statistical Manual of Mental Disorders IV criteria; 5) schizophrenia history $\leq 5$ years; 6 ) ability to sign informed consent; 7) single antipsychotic usage for at least 4 weeks before study, reaching at least minimum treatment dosage; 8) as per researchers' judgment: clinical global impression of severity (CGI-S) score changes $\leq 1$ for at least 4 weeks before study, no obvious aggressive behavior, lack of cooperation or apparent restlessness, ability to complete the 2-hour examination; 9) dissatisfaction with efficacy or lack of treatment before the study with a positive and negative syndrome scale (PANSS) total score $\geq 70$, or more than one item rated $>4$ on either the positive or negative symptom subscale, or more than two items rated $>4$ on the general psychopathology subscale.

Exclusion criteria included the following: alcohol or substance dependence in the past 3 months; significant clinical neural disease; history of head trauma (loss of consciousness $>1$ hour); any current medical condition that would interfere with the assessment of efficacy; symptoms of acute deterioration such as aggravated illness requiring hospitalization or increased intensive mental rehabilitation care; significant muscle tension or Parkinson's disease; clinically significant abnormal laboratory test results (blood, urine, and blood biochemical analysis); abnormal electrocardiogram as judged by researchers; pregnant or nursing females; and those undergoing treatment with clozapine, cognitive enhancement agents (methylphenidate), antidementia drugs, amphetamines, lithium salts, monoamine oxidase inhibitors, combinations of QTc-prolonging drugs 
(male: QTc $>450 \mathrm{~mm}$, female: QTc $>470 \mathrm{~mm}$ ) such as sotalol, quinidine, amiodarone, erythromycin, or tricyclic antidepressants. Participants using benzodiazepines, sedative drugs, or anticholinergic agents within 12 hours of the assessment, those who had undergone electroconvulsive therapy within 1 month of the study, those who had received longacting injection treatment within 1 month of the study, those who could not swallow medication with water (chewing, breaking, dissolving, or mashing research drugs was not allowed, as these may affect the release of drugs), and those with allergic reaction to paliperidone or risperidone were also excluded from the present study.

\section{Treatment}

Upon initiation of paliperidone ER treatment, patients were tapered off their previous oral antipsychotics within a maximum of 4 weeks. The dosage of paliperidone ER was flexible and ranged from 3 to $12 \mathrm{mg}$ /day during the 24-week study period. Assessments were performed on day 1 and then every 4 weeks until the conclusion of the study period. Assessment data, adverse events (AEs), and concomitant medications were consistently recorded during this period (Figure 1). Use of antidepressants, mood stabilizers, cognitive enhancement drugs, or antidementia drugs was not permitted. Benzodiazepines were not permitted within 12 hours of assessment with the measurement and treatment research to improve cognition in schizophrenia (MATRICS) consensus cognitive battery.

\section{Research methods}

\section{Assessment tools}

PSP scale

The PSP scale evaluates the severity of the patient's condition across four domains: socially useful activities, personal and social relationships, self-care, and disturbing/aggressive behavior in the past month. The individual subscale scores are added together, with a total possible score of 100. Patients are assigned one of ten possible grades of severity. ${ }^{11}$

\section{MATRICS consensus cognitive battery}

The MATRICS consensus cognitive battery (MCCB) assesses functioning in seven cognitive domains: attention, information processing speed, verbal learning and memory, visual learning and memory, working memory, reasoning, and problem-solving, and social cognition. The US norm of MCCB was developed by Kern et al. ${ }^{12}$ A Chinese version of this assessment with high reliability and validity was created and further revised by Shi et al. ${ }^{13}$ All examiners

\begin{tabular}{|c|c|c|c|c|c|c|c|}
\hline Visit content & $\begin{array}{l}\text { Visit } 1 \\
\text { screening } \\
\text { and baseline }\end{array}$ & $\begin{array}{l}\text { Visit } 2^{\mathrm{b}} \\
28 \pm 3 \text { days } \\
(4 \text { weeks) }\end{array}$ & $\begin{array}{l}\text { Visit } 3 \\
56 \pm 3 \text { days } \\
\text { (8 weeks) }\end{array}$ & $\begin{array}{l}\text { Visit } 4 \\
84 \pm 3 \text { days } \\
\text { (12 weeks) }\end{array}$ & $\begin{array}{l}\text { Visit } 5 \\
112 \pm 5 \text { days } \\
\text { (16 weeks) }\end{array}$ & $\begin{array}{l}\text { Visit } 6 \\
140 \pm 5 \text { days } \\
\text { (20 weeks) }\end{array}$ & $\begin{array}{l}\text { Visit } 7^{\mathrm{a}} \\
168 \pm 5 \text { days } \\
\text { (24 weeks) } \\
\text { endpoint }\end{array}$ \\
\hline Informed consent ${ }^{c}$ & $x$ & & & & & & \\
\hline Inclusion/exclusion criteria & $x$ & & & & & & \\
\hline Demographic and clinical data & $x$ & & & & & & \\
\hline Study drug and record dose & $X^{d}$ & $X^{e}$ & $x^{e}$ & $x^{e}$ & $X^{e}$ & $X^{e}$ & $X^{e}$ \\
\hline MATRICS-MCCB & $x$ & & & & & & $x$ \\
\hline PANSS & $x$ & $x$ & $x$ & $x$ & $x$ & $x$ & $x$ \\
\hline CGI-S & $x$ & $x$ & $x$ & $x$ & $x$ & $x$ & $x$ \\
\hline PSP & $x$ & $x$ & & $x$ & & & $x$ \\
\hline Vital signs inspection & $x$ & & & & & & $x$ \\
\hline AIMS/BARS/SAS & $x$ & & & & & & $x$ \\
\hline Lab tests ${ }^{f}$ & $x$ & & & & & & $x$ \\
\hline Electrocardiogram & $x$ & & & & & & $x$ \\
\hline Serum ChE activity & $x$ & & & & & & $x$ \\
\hline Concomitant therapyg & $x$ & $x$ & $x$ & $x$ & $x$ & $x$ & $x$ \\
\hline Adverse event & $x$ & $x$ & $x$ & $x$ & $x$ & $x$ & $x$ \\
\hline
\end{tabular}

Figure I Research flow chart.

Notes: aBaseline survey should be before the change of medication. If the test is terminated prematurely, endpoint visit program should be done by the termination of the test. ' $T$ The previously used antipsychotic drugs have been completely swapped with paliperidone ER tablets. Informed consent of participants should be obtained before any research-related programs. ${ }^{d}$ Baseline dose is the prescription dose by researchers in follow-up treatment with subjects. ${ }^{\mathrm{e}}$ Follow-up and endpoint dose record is the actual dose taken by patients. flaboratory tests include blood and urine tests, liver function (ALT, AST, bilirubin), renal function (creatinine, blood urea nitrogen), and lipids

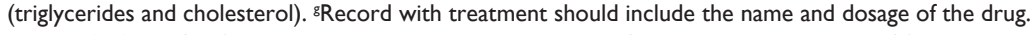

Abbreviations: AIMS, abnormal involuntary movement scale; ALT, alanine transaminase; AST, aspartate aminotransferase; BARS, Barnes akathisia scale; CGI-S, clinical global impression-severity; ER, extended-release; MATRICS, measurement and treatment research to improve cognition in schizophrenia; MCCB, MATRICS consensus cognitive battery; PANSS, positive and negative syndrome scale; PSP, personal and social performance; SAS, Simpson-Angus scale. 
who conducted MCCB assessments were trained and certified by the MATRICS group from the US.

\section{Positive and negative syndrome scale}

The PANSS includes 30 items designed to assess positive (seven items), negative (seven items), and general psychopathology (16 items) symptoms in patients with schizophrenia. Participants respond by rating the degree of severity for specific items according to the following: 1-absent, 2-minimal, 3-mild, 4-moderate, 5-moderate-severe, 6-severe, and 7-extreme. ${ }^{14}$

\section{Clinical global impression of severity}

The CGI-S rating scale is commonly used to assess symptom severity. The CGI-S evaluates the overall condition of patients within a given time on a 7-point scale, with 1 indicating normal health/absence of illness and 7 indicating extreme illness. ${ }^{15}$

\section{Extrapyramidal symptom rating scales: abnormal involuntary movement scale, Barnes akathisia scale, and Simpson-Angus scale}

The abnormal involuntary movement scale (AIMS includes 12 items associated with face and mouth movements, body movement, trunk movement, and overall symptoms, rated along a scale from 0 (normal) to 4 (severe). ${ }^{16}$ The Barnes akathisia scale (BARS contains one objective assessment and two subjective assessments (the subjective awareness of restlessness, and any distress associated with akathisia) for rating observable restless movements from 0 (light) to 3 (severe). ${ }^{17}$ The BARS also includes an overall assessment of restless movements, rated from 0 (absent) to 5 (severe). The Simpson-Angus scale (SAS assesses the following ten areas, rated from 0 (normal) to 4 (severe): gait, arm dropping, shoulder shaking, elbow rigidity, wrist stiffness, leg swinging, head rotation, glabella tap, tremor, and salivation. ${ }^{18}$

AEs were recorded during each follow-up visit. Vital signs, urine function, liver and kidney function, serum cholinesterase activity, and electrocardiogram data were also recorded at baseline and upon conclusion of the study period.

\section{Statistical analysis}

\section{Analysis of data sets}

\section{Full analysis set}

According to the basic principle of intentionality analysis, all patients who received paliperidone ER treatment at least once during the study period and at least one treatment evaluation after baseline were included in the full analysis set (FAS).
The missing data in the main outcome measure of FAS were filled using the last observation carried forward method.

Per protocol set

Per protocol set (PPS) is a subset of the FAS, which refers to patients with no serious violations in the FAS research program and good compliance ( $\geq 80 \%$ days of proper medication), and no missing data of the main outcome measure. PPS was used as the primary efficacy analysis of this study.

Safety analysis (safety set)

All those who received at least one paliperidone ER tablet during the treatment period and one safety evaluation were included in the safety set (SS) of the study. The SS served as the security analysis of this study.

\section{Statistical methods}

Descriptive statistical analysis was conducted for demographic information, baseline characteristics, assessment scores, and AEs (Table 1). The mean, standard deviation, effective number, median, maximum, and minimum values, and $95 \%$ confidence interval of the mean were calculated for all data. The frequency, percentage, and 95\% confidence interval of the percentage were also calculated. A paired $t$-test or Wilcoxon rank-sum test was used for within groupgroup analysis based on the normality of the distribution. Two-tailed tests with a significance level of $P<0.05$ were

Table I Demographic and baseline data of full analysis set (FAS, $n=90$ )

\begin{tabular}{ll}
\hline Items & $\begin{array}{l}\text { Mean } \pm \text { SD (95\% Cl) } \\
\text { or } \mathbf{n}(\%)\end{array}$ \\
\hline $\begin{array}{l}\text { Age (years) } \\
\text { Sex, } \mathrm{n}(\%)\end{array}$ & $27.56 \pm 7.75(18.00-45.00)$ \\
$\quad$ Male & $44(48.89)$ \\
$\quad$ Female & $46(51.11)$ \\
Ethnicity, $\mathrm{n}(\%)$ & \\
$\quad$ Han & $89(98.89)$ \\
Marital status, $\mathrm{n}(\%)$ & \\
$\quad$ Married & $17(18.89)$ \\
$\quad$ Unmarried & $68(75.56)$ \\
$\quad$ Other & $5(5.55)$ \\
Family history, $\mathrm{n}(\%)$ & \\
$\quad$ Yes & $12(13.33)$ \\
$\quad$ No & $78(86.67)$ \\
Schizophrenia type, $\mathrm{n}$ (\%) & \\
$\quad$ Paranoid & $60(66.67)$ \\
$\quad$ Undifferentiated & $27(30.00)$ \\
$\quad$ Others & $3(3.33)$ \\
Duration of illness (years) & $2.18 \pm 1.63(0.00-5.53)$ \\
Duration of untreated psychosis (months) & $9.80 \pm 12(0.00-53.00)$ \\
Frequency of relapse & $1.79 \pm 1.96(0.00-10.00)$ \\
Frequency of hospitalization & $1.07 \pm 1.31(0.00-6.00)$ \\
\hline Abbrevians: Cl, &
\end{tabular}

Abbreviations: $\mathrm{Cl}$, confidence interval; FAS, full analysis set. 
performed, and Bonferroni's method was used in the correction of multiple comparisons.

\section{Sample size calculation}

According to the results of the previous studies, we assumed a standard deviation of 13.5, along with an average difference of 6.4 in PSP scores between baseline and poststudy assessment. ${ }^{19}$ Without the medication change intervention, the estimated PSP score is expected to improve by two points. ${ }^{20}$ Therefore, a 0.025 probability of class I errors ( single side) with $80 \%$ confidence could be expected in 76 participants. Considering a $20 \%$ test failure rate, the sample size required for statistically significant conclusions should be 95 participants.

\section{Results}

In the present study, we enrolled 95 participants, two of whom were excluded from the statistical analyses (SS, FAS, and PPS) due to lack of posttreatment safety and medication data. Therefore, 93 (97.89\%) participants were included in the SS. Two participants declined to undergo the efficacy evaluation after treatment, and one patient became pregnant during the study period. These three cases were also excluded from the FAS, which included a total of $90(94.74 \%)$ cases (Table 1$)$. The average dosage of paliperidone ER was $7.44 \pm 2.89 \mathrm{mg}$ per day. Medications taken by participants prior to the study period are listed in Table 2 . Thirty-five patients (38.90\%) were switched from risperidone, while 18 were switched from olanzapine $(20.00 \%)$. Fifteen participants dropped out during the study period, while three participants exceeded the time allowed for follow-up assessment. Therefore, 72 (75.79\%) participants were included in the PPS.

Figure 2 and Table 3 depict data regarding the primary outcome of improvement in social function. The mean PSP score was $54.3 \pm 14.3$ at baseline, which significantly increased to $73.4 \pm 12.6$ at week $24(P<0.001, t$-test $)$, with an increase of $19.1 \pm 16.1$. The percentage of patients with PSP scores over 70 points was $65.3 \%$. Changes in PSP score from

Table 2 Prestudy medications

\begin{tabular}{lll}
\hline Medication & $\begin{array}{l}\text { Number of } \\
\text { patients }(\%)\end{array}$ & $\begin{array}{l}\text { Dosage range } \\
\text { (mg/day) }\end{array}$ \\
\hline Risperidone & $35(38.90)$ & $1-6$ \\
Olanzapine & $18(20.00)$ & $2.5-30$ \\
Aripiprazole & $14(15.56)$ & $5-30$ \\
Quetiapine & II (12.22) & $400-600$ \\
Ziprasidone & $4(4.44)$ & $40-120$ \\
Perphenazine & $3(3.33)$ & $4-40$ \\
Sulpiride & $3(3.33)$ & $200-900$ \\
Amisulpride & $2(2.22)$ & $400-800$ \\
\hline
\end{tabular}

baseline $\geq 10$ points were observed in $77.8 \%$ of participants. The percentage of patients with mild or no functional impairment as indicated by PSP scores increased relative to baseline for all domains. The greatest improvement was observed in the individual domain of personal and social relationships (no-to-mild functional impairment in $12.22 \%$ of participants at baseline and $63.33 \%$ at endpoint), followed by socially useful activities (no-to-mild functional impairment in 14.44\% of participants at baseline and 55.56\% at endpoint).

We also assessed the improvement of neurocognitive function using the MCCB. Significant changes were observed in six of the nine individual raw subtest scores $(P<0.05$, paired $t$-test or sign-rank test) between baseline and endpoint (week 24): Trail making test: Part A, Hopkins verbal learning test-revised, spatial span, mazes, brief visuospatial memory test-revised, and continuous performance test-identical pairs. Significant changes were observed in six of seven cognitive domains, including processing speed, verbal learning, working memory, attention/vigilance, visual learning, reasoning and problem-solving, as well as in total cognitive $t$ scores $(P<0.001$, paired $t$-test or sign-rank test) between baseline and endpoint. As shown in Table 4 and Figure 3, the effect on overall cognitive function improvement as indicated by Cohen's d was $=0.47, P<0.001$.

In terms of psychiatric symptom improvement, analysis of PANSS scores indicated that, from the beginning of the fourth week, PANSS total scores, PANSS positive symptoms scores, PANSS negative symptoms scores, and PANSS general pathology scores $(62.53 \pm 14.35,13.67 \pm 5.72,18.01 \pm 5.91$, and $30.85 \pm 6.16$, respectively) decreased significantly $(P<0.01)$ compared with baseline scores (75.24 $\pm 15.31,17.46 \pm 7.01$, $20.96 \pm 6.07$, and 36.82 \pm 6.86 , respectively). Scores continued to improve until the endpoint of the treatment period $(46.54 \pm 13.82,9.63 \pm 4.67,13.57 \pm 5.45$, and $24.14 \pm 5.17$, respectively) (Figure 4). The same trend was also observed for CGI-S scores at baseline, week 4, and treatment endpoint: 4.67 \pm 0.90 , $3.93 \pm 0.95$, and $2.83 \pm 1.02$, respectively (Figure 5).

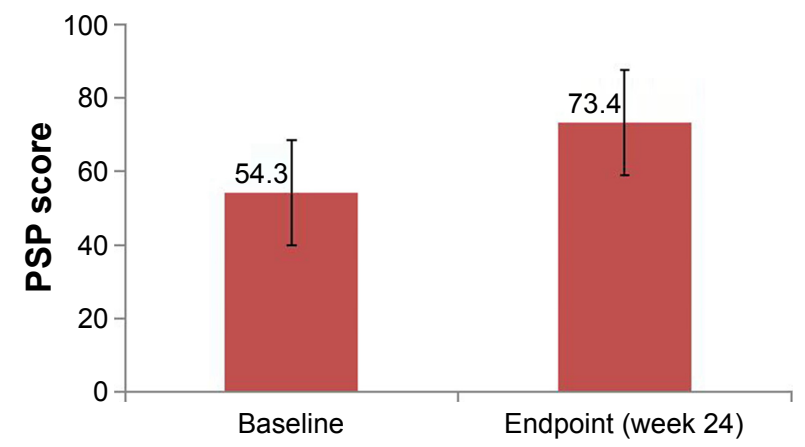

Figure 2 Social function improvement after 24 weeks of treatment with paliperidone. Abbreviation: PSP, personal and social performance. 
Table 3 Proportion of participants exhibiting changes in PSP scores in each dimension at each visit

\begin{tabular}{|c|c|c|c|c|}
\hline \multirow[t]{2}{*}{ PSP } & \multicolumn{4}{|c|}{$\operatorname{FAS}^{\mathrm{a}}(\mathrm{n}=90)$} \\
\hline & Baseline & Week 4 & Week I2 & Week 24 \\
\hline \multicolumn{5}{|c|}{ Socially useful activities, including work and study, n (\%) } \\
\hline Absent & $3(3.33)$ & $3(3.33)$ & $4(4.44)$ & $7(7.78)$ \\
\hline Mild & $10(11.11)$ & $15(16.67)$ & $25(27.78)$ & $43(47.78)$ \\
\hline Manifest & $28(31.1 I)$ & $50(55.56)$ & $49(54.45)$ & $33(36.66)$ \\
\hline Marked & $34(37.78)$ & $20(22.22)$ & $\mathrm{II}(\mathrm{I} 2.22)$ & $6(6.67)$ \\
\hline Severe & $15(16.67)$ & $2(2.22)$ & $\mathrm{I}(\mathrm{I} . \mathrm{II})$ & $\mathrm{I}(\mathrm{I} . \mathrm{II})$ \\
\hline Very severe & $0(0.00)$ & $0(0.00)$ & $0(0.00)$ & $0(0.00)$ \\
\hline$P$-value ${ }^{b}$ & & $<0.000$ I & $<0.0001$ & $<0.000 \mathrm{I}$ \\
\hline \multicolumn{5}{|c|}{ Personal and social relationships, $\mathrm{n}(\%)$} \\
\hline Absent & $\mathrm{I}(\mathrm{I} . \mathrm{II})$ & $2(2.22)$ & $4(4.44)$ & $10(I I . I I)$ \\
\hline Mild & $10(11.11)$ & $21(23.33)$ & $33(36.67)$ & $47(52.22)$ \\
\hline Manifest & $35(38.90)$ & $49(54.45)$ & $46(5 I .11)$ & $26(28.90)$ \\
\hline Marked & 31 (34.44) & $13(14.44)$ & $5(5.56)$ & $4(4.44)$ \\
\hline Severe & $13(14.44)$ & $5(5.56)$ & $2(2.22)$ & $3(3.33)$ \\
\hline Very severe & $0(0.00)$ & $0(0.00)$ & $0(0.00)$ & $0(0.00)$ \\
\hline$P$-value ${ }^{b}$ & & $<0.0001$ & $<0.0001$ & $<0.0001$ \\
\hline \multicolumn{5}{|l|}{ Self-care, n (\%) } \\
\hline Absent & $22(24.44)$ & $34(37.78)$ & $43(47.78)$ & $54(60.00)$ \\
\hline Mild & $30(33.33)$ & $39(43.33)$ & $36(40.00)$ & $27(30.00)$ \\
\hline Manifest & $24(26.67)$ & $14(15.56)$ & $9(10.00)$ & $7(7.78)$ \\
\hline Marked & 14 (I5.56) & $3(3.33)$ & $2(2.22)$ & $2(2.22)$ \\
\hline Severe & $0(0.00)$ & $0(0.00)$ & $0(0.00)$ & $0(0.00)$ \\
\hline Very severe & $0(0.00)$ & $0(0.00)$ & $0(0.00)$ & $0(0.00)$ \\
\hline$P$-value ${ }^{b}$ & & $<0.0001$ & $<0.0001$ & $<0.0001$ \\
\hline \multicolumn{5}{|c|}{ Disturbing and aggressive behaviors, $n$ (\%) } \\
\hline Absent & $45(50.00)$ & $58(64.44)$ & 69 (76.67) & 7I (78.89) \\
\hline Mild & $27(30.00)$ & $24(26.67)$ & $12(13.33)$ & $10(11.11)$ \\
\hline Manifest & $8(8.88)$ & $7(7.78)$ & $8(8.89)$ & $8(8.89)$ \\
\hline Marked & $5(5.56)$ & $I(I . I I)$ & I (I.II) & I (I.II) \\
\hline Severe & $5(5.56)$ & $0(0.00)$ & $0(0.00)$ & $0(0.00)$ \\
\hline Very severe & $0(0.00)$ & $0(0.00)$ & $0(0.00)$ & $0(0.00)$ \\
\hline$P$-value ${ }^{b}$ & & - & - & - \\
\hline
\end{tabular}

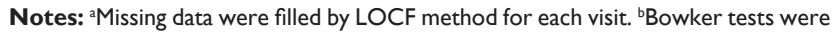
used to analyze the change after treatment and calculate $P$-value.

Abbreviations: FAS, full analysis set; LOCF, last observation carried forward; PSP, personal and social performance.
A multivariate regression model was established to explore the factors affecting functional outcomes, using PSP improvement as the dependent variable, while MCCB and PANSS score improvement, and AIMS, SAS, and BARS scores and serum cholinesterase changes were regarded as independent variables. The results of this analysis indicated that the MCCB verbal learning and PANSS scores should be entered into the regression equation, with partial regression coefficients of $0.34(P<0.001)$ and $0.67(P<0.05)$, respectively.

No significant difference was observed $(P>0.05)$ in vital signs, serum cholinesterase, AIMS score, SAS score, and BARS score between the study endpoint (24 weeks) and baseline in SS.

AEs occurred in $62.4 \%$ of patients: Fifty-eight patients experienced a total of 129 AEs, most of which were mild (44.1\%) and moderate (32.3\%) in severity (Table 5). Two severe AEs (2.2\%) included cardiac arrest and an accidental pregnancy. Fifty-four participants $(58.1 \%)$ experienced possible treatment-emergent adverse events. The most common AEs (incidence $\geq 5 \%$ ) were extrapyramidal symptoms (34.4\%), body weight gain (14.0\%), amenorrhea (7.5\%), anxiety (5.4\%), and dysphoria (3.2\%). All treatmentemergent adverse events reported in $\geq 2 \%$ of the patients are listed in Table 5.

\section{Discussion}

We observed that paliperidone ER treatment was associated not only with significant improvement in positive, negative, and general pathological symptoms in patients with schizophrenia but also with comprehensive improvement

Table 4 Improvements in neurocognitive test scores after 24 weeks of treatment with paliperidone (raw scores)

\begin{tabular}{|c|c|c|c|c|c|c|c|}
\hline \multirow[t]{2}{*}{ Subtests } & \multicolumn{2}{|l|}{ Baseline } & \multicolumn{2}{|l|}{ Endpoint } & \multirow[t]{2}{*}{$\mathbf{Z} / \mathbf{t}$} & \multirow[t]{2}{*}{$P$-value } & \multirow{2}{*}{$\begin{array}{l}\text { Cohen's } \\
\text { d }\end{array}$} \\
\hline & $x \pm S D$ & $95 \% \mathrm{Cl}$ & $\mathbf{x} \pm \mathbf{S D}$ & $95 \% \mathrm{Cl}$ & & & \\
\hline Trail making test: part $\mathrm{A}$ & $48.1 I \pm 23.48$ & $42.5-53.63$ & $40.24 \pm 17.67$ & $36.08-44.39$ & -640.00 & $<0.0001$ & 0.38 \\
\hline $\begin{array}{l}\text { Brief assessment of cognition in } \\
\text { schizophrenia: symbol coding }\end{array}$ & $44.88 \pm 10.82$ & $42.33-47.42$ & $46.58 \pm 11.37$ & $43.91-49.26$ & 225.00 & 0.1610 & 0.15 \\
\hline $\begin{array}{l}\text { Hopkins verbal learning } \\
\text { test-revised }\end{array}$ & $22.32 \pm 6.36$ & $20.82-23.81$ & $24.69 \pm 5.52$ & $23.40-25.99$ & 4.69 & $<0.000$ I & 0.40 \\
\hline $\begin{array}{l}\text { Wechsler memory scale-third } \\
\text { edition: spatial span }\end{array}$ & $14.79 \pm 3.30$ & $14.02-15.57$ & $15.88 \pm 3.56$ & $|5.04-| 6.7 \mid$ & 445.00 & 0.0006 & 0.32 \\
\hline $\begin{array}{l}\text { Neuropsychological assessment } \\
\text { battery: mazes }\end{array}$ & $|3.08 \pm 6.5|$ & $|1.55-| 4.6 \mid$ & $|4.89 \pm 6.3|$ & $13.4 \mid-16.37$ & 2.81 & 0.0063 & 0.28 \\
\hline $\begin{array}{l}\text { Brief visuospatial memory } \\
\text { test-revised }\end{array}$ & $22.47 \pm 7.76$ & $20.65-24.30$ & $25.63 \pm 7.31$ & $23.91-27.34$ & 4.55 & $<0.000$ I & 0.42 \\
\hline $\begin{array}{l}\text { Category fluency test: animal } \\
\text { naming (fluency) }\end{array}$ & $17.13 \pm 5.67$ & $15.79-18.46$ & $17.64 \pm 5.32$ & $16.39-18.89$ & 1.05 & 0.2972 & 0.09 \\
\hline $\begin{array}{l}\text { Mayer-Salovey-Car emotional } \\
\text { intelligence test: managing emotions }\end{array}$ & $78.09 \pm 8.06$ & $76.20-79.99$ & $78.19 \pm 9.18$ & $75.98-80.39$ & 0.21 & 0.8366 & 0.01 \\
\hline $\begin{array}{l}\text { Continuous performance } \\
\text { test-identical pairs }\end{array}$ & $2.07 \pm 0.78$ & $1.88-2.25$ & $2.20 \pm 0.70$ & $2.04-2.37$ & 2.19 & 0.0316 & 0.18 \\
\hline
\end{tabular}

Abbreviations: $\mathrm{Cl}$, confidence interval; SD, standard deviation. 


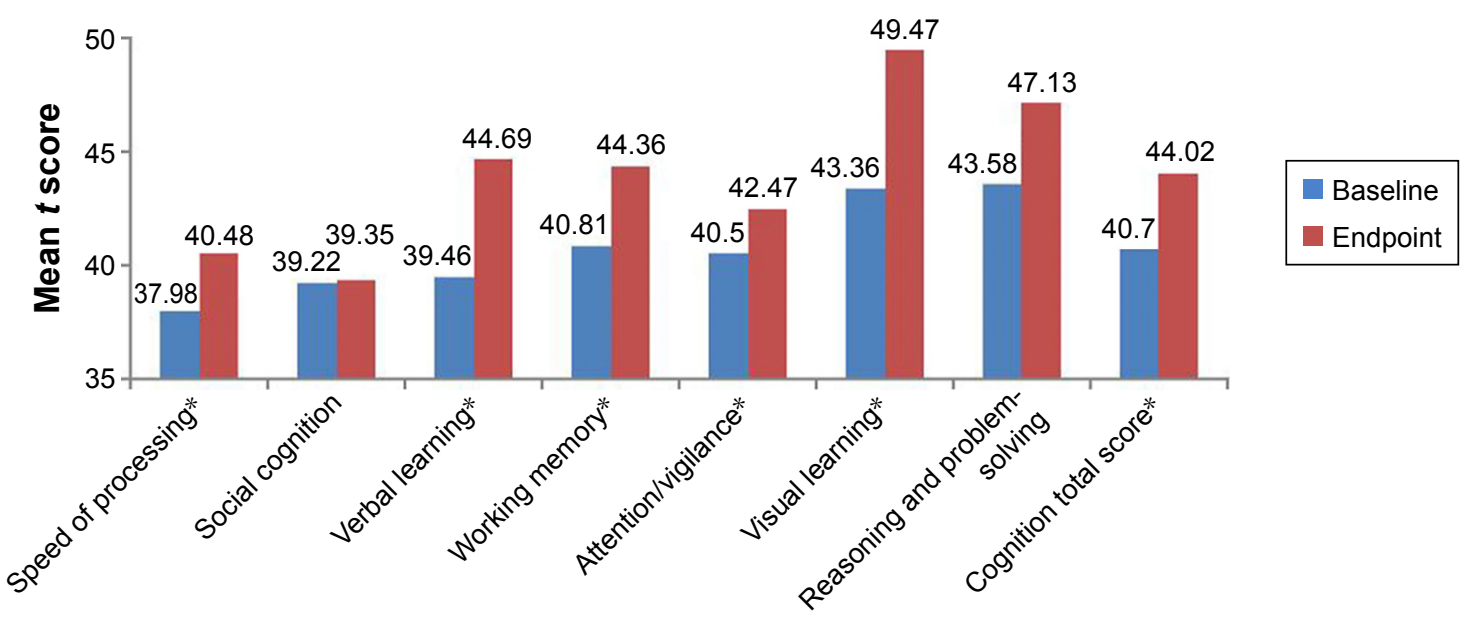

Figure 3 Neurocognitive improvements after 24 weeks of treatment with paliperidone ( $t$ score).

Note: *Compared to baseline, $P<0.001$, paired $t$-test or signed-rank test.

in cognitive function as well as functional outcomes. Paliperidone ER treatment was associated with fewer and more mild adverse reactions in safety assessment, supporting its use in the treatment of schizophrenia.

Functional evaluation is difficult in patients with schizophrenia because it requires the individual to respond to realworld situations. Functional assessment methods usually involve a combination of self-assessment, source assessment, interview, observation, and evaluation, though interviews are the most common psychiatric clinical methods. ${ }^{21}$ Global assessment of function and PSP are the most commonly used interview-based assessments. The PSP has been validated in the People's Republic of China, suggesting that PSP has better psychometric characteristics and intercultural effectiveness in Chinese patients with schizophrenia. ${ }^{22}$ In this study, the Chinese version of PSP exhibited good internal consistency (Cronbach's alpha $=0.84$ ), good interrater reliability (kappa value $=0.82$, intraclass correlation coefficient $=0.94$ ), and good retest reliability (intraclass correlation coefficient $=0.95$ ), proving PSP to be a convenient, valid, and reliable instrument for assessing function in Chinese patients with schizophrenia. Many factors affect functional outcomes in schizophrenia: Sex, level of education, social support, sense of shame, positive symptoms, negative symptoms, cognitive function, depressive symptoms, premorbid level of functioning, and duration of untreated psychosis, all exert influence on individual functional outcomes. ${ }^{23-27}$ Our study showed that the percentage of participants with PSP scores $>70$ points had increased to $65.3 \%$ and that approximately two-thirds of participants experienced substantial improvement, indicating the efficacy of long-term paliperidone treatment for schizophrenia. PANSS total scores, which most significantly reflect functional outcomes via assessment of overall psychotic symptoms and verbal learning skills, also

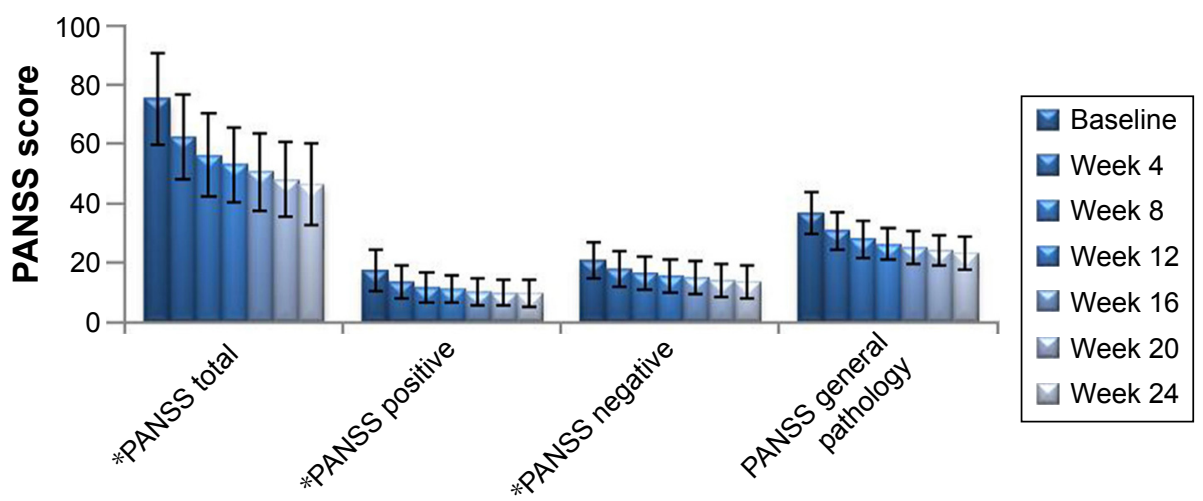

PANSS score (mean \pm SD)

Figure 4 Changes in PANSS total score after treatment with paliperidone.

Note: *Compared to baseline, $P<0.001$ since week 4 , paired $t$-test or signed-rank test.

Abbreviations: PANSS, positive and negative syndrome scale; SD, standard deviation. 


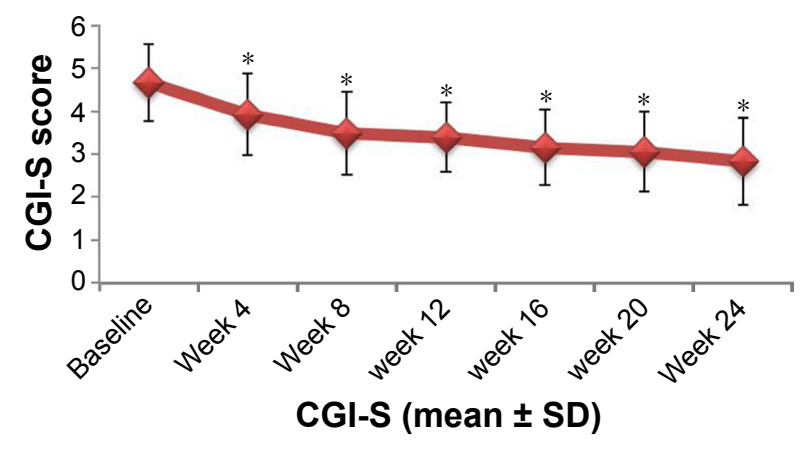

Figure 5 Changes in CGI-S score after treatment with paliperidone. Note: *Compared to baseline, $P<0.001$ since week 4 , paired $t$-test or signed-rank test. Abbreviations: $\mathrm{CGI}-\mathrm{S}$, clinical global impression-severity; SD, standard deviation.

improved in this study. This is consistent with previous research suggesting that verbal learning and memory skills were predictors of better functional outcomes, especially social problem-solving ability. ${ }^{28}$

Impaired cognitive function is one of the core symptoms of schizophrenia, which occurs in patients with abnormalities in brain function and structure, as well as those with genetic, neural, and biochemical abnormalities, rendering comprehensive treatment difficult. Currently, the treatment of impaired cognition due to schizophrenia with antipsychotic drugs is not promising. Meta-analyses of randomized double-blind controlled experiments reported that the most significant improvements occurred with regard to attention, visual-spatial abilities, and executive function, and that the overall efficacy was poor, with an average Cohen's

Table 5 Treatment-emergent adverse events

\begin{tabular}{|c|c|}
\hline TEAE & $\begin{array}{l}\text { Patients } \\
(\mathbf{N}=93) \\
\mathrm{n}(\%)\end{array}$ \\
\hline Severity & $58(62.37)$ \\
\hline NA & I (I.08) \\
\hline Mild & $4 \mid(44.09)$ \\
\hline Moderate & $30(32.26)$ \\
\hline Severe & $3(3.23)$ \\
\hline \multicolumn{2}{|c|}{ TEAEs occurring in $\geq$ EAEs occurring } \\
\hline Extrapyramidal symptoms & $32(34.4 I)$ \\
\hline Weight gain & $13(13.98)$ \\
\hline Amenorrhea & $7(7.53)$ \\
\hline Anxiety & $5(5.38)$ \\
\hline Akathisia & $3(3.23)$ \\
\hline Insomnia & $3(3.23)$ \\
\hline Headache & $3(3.23)$ \\
\hline Dysphoria & $3(3.23)$ \\
\hline Blood prolactin abnormal & $2(2.15)$ \\
\hline Dizziness & $2(2.15)$ \\
\hline Inappropriate affect & $2(2.15)$ \\
\hline Depression & $2(2.15)$ \\
\hline Constipation & $2(2.15)$ \\
\hline Nausea & $2(2.15)$ \\
\hline
\end{tabular}

Abbreviations: EAE, emergent adverse events; TEAE, treatment-emergent adverse events; NA, not available. $\mathrm{d}=0.22 .{ }^{29-31}$ Previous studies have demonstrated improvements in cognition in patients with schizophrenia after treatment with atypical antipsychotics such as risperidone and clozapine. ${ }^{32}$ Though the effects of paliperidone are yet to be verified with regard to cognitive improvements, paliperidone ER treatment in the present study resulted in significant improvements in six of seven MCCB domains following treatment. The overall improvement effect value was 0.47 , indicating a moderate effect. To our knowledge, our study is the first to demonstrate significant improvements in cognition, as assessed by MCCB, in Chinese patients with schizophrenia undergoing paliperidone ER treatment. These results may help to shed light on the treatment of impaired cognition in Chinese patients with schizophrenia.

Extrapyramidal reactions remain the most common adverse effect associated with paliperidone treatment. Nearly one-third of patients exhibited extrapyramidal symptoms, though the overall severity of the symptoms was moderate. Weight problems cannot be neglected, although the incidence rate in our study was only $14 \%$. However, weight gain places patients at risk for metabolic syndrome, and obesity also reduces the quality of life. ${ }^{33}$ Amenorrhea was not a very prominent problem. Overall, the results of the present study support the idea that long-term treatment with paliperidone is safe yet effective.

The present study has three limitations. First, the openlabel and single-arm design of this study, wherein patients were aware that they were being treated with active products, may have resulted in bias regarding efficacy or the reporting of AEs. However, this study was an exploratory trial aimed at investigating the effects of paliperidone ER on neurocognitive and social function. Second, we used the same version of the MCCB tool to assess verbal and visual learning both before and after the treatment. Although the assessments were conducted 6 months apart, this may be another confounding factor with regard to the results of the study. Finally, no functional magnetic resonance imaging or genetic studies have been conducted to further clarify the mechanism underlying improvements in cognitive function associated with paliperidone ER. Therefore, future randomized, double-blind, controlled studies incorporating both behavioral and imaging assessments are required in order to more fully evaluate improvements in neurocognitive and social function due to paliperidone ER treatment.

\section{Conclusion}

Treatment with paliperidone ER significantly improves social and neurocognitive function as well as symptoms in Chinese patients with schizophrenia. 


\section{Acknowledgments}

This research is an investigator initiated study (R076477SCH4048) supported by Xian Janssen Pharmaceutical Company.

This research was supported in part by the National Key Project of Scientific and Technical Supporting Programs Funded by Ministry of Science \& Technology of China (No 2007BAI17B04), Beijing Ministry of Science \& Technology of China Supporting Programs Funded by Ministry of Science \& Technology of Beijing (No D121100005012004).

The study team gratefully acknowledges the assistance of Robert K. Heaton, who provided many useful suggestions in the development of the study protocol; and Kang Lan, Tang Xirong, Peng Daihui, Lin Huali, Liu Fang, and Wang Xijin, who completed the neuropsychological and functioning assessments. The authors thank the patients for their participation in the study.

\section{Author contributions}

$\mathrm{CS}, \mathrm{XY}, \mathrm{SQY}$, and HJ designed the study and wrote the protocol. CS managed the literature searches and analyses. CS, SQY, YFX, JGS, XFX, and CPZ selected the sample, evaluated the patients, and contributed in some aspects of the study design and in the interpretation of results. CS undertook the statistical analyses. CS, XY, and HJ wrote the first draft of the manuscript. All authors contributed toward data analysis, drafting and revising the paper and agree to be accountable for all aspects of the work.

\section{Disclosure}

The authors declared that they have no commercial or associative interest that represents a conflict of interest in connection with Xian Janssen Pharmaceutical Ltd. Xian Janssen Pharmaceutical Ltd did not undertake any responsibility in the design and drafting of the protocol, monitoring of the study, provision of data management and analysis, identification of the sponsor -investigator and study participants, or selection of study personnel. The authors report no conflicts of interest in this work.

\section{References}

1. de Leon J, Wynn G, Sandson NB. The pharmacokinetics of paliperidone versus risperidone. Psychosomatics. 2010;51(1):80-88.

2. Charnsil C, Vongpanich S. An open-label, prospective study to evaluate social function and overall improvement of extended-release paliperidone treatment in Thai schizophrenia patients. Neuropsychiatr Dis Treat. 2013;9:1223-1230

3. Gattaz WF, Campos JA, Lacerda AL, et al. Switching from oral risperidone to flexibly dosed oral paliperidone extended-release: core symptoms, satisfaction, and quality of life in patients with stable but symptomatic schizophrenia: the RISPALI study. Curr Med Res Opin. 2014;30(4):695-709.
4. Nakagawa R, Ohnishi T, Kobayashi H, et al. The social functional outcome of being naturalistically treated with paliperidone extendedrelease in patients with schizophrenia. Neuropsychiatr Dis Treat. 2015; 11:1511-1521.

5. Zhang L, Li J, Zhao Y, Su Y, Si T. Critical evaluation of paliperidone in the treatment of schizophrenia in Chinese patients: a systematic literature review. Neuropsychiatr Dis Treat. 2016;12:113-131.

6. Houthoofd SA, Morrens M, Sabbe BG. Cognitive and psychomotor effects of risperidone in schizophrenia and schizoaffective disorder. Clin Ther. 2008;30(9):1565-1589.

7. Harvey PD, Patterson TL, Potter LS, Zhong K, Brecher M. Improvement in social competence with short-term atypical antipsychotic treatment: a randomized, double-blind comparison of quetiapine versus risperidone for social competence, social cognition, and neuropsychological functioning. Am J Psychiatry. 2006;163(11):1918-1925.

8. Sergi MJ, Green MF, Widmark C, et al. Social cognition [corrected] and neurocognition: effects of risperidone, olanzapine, and haloperidol. Am J Psychiatry. 2007;164(10):1585-1592.

9. Maat A, Cahn W, Gijsman HJ, Hovens JE, Kahn RS, Aleman A. Open, randomized trial of the effects of aripiprazole versus risperidone on social cognition in schizophrenia. Eur Neuropsychopharmacol. 2014; 24(4):575-584.

10. Kim SW, Chung YC, Lee YH, et al. Paliperidone ER versus risperidone for neurocognitive function in patients with schizophrenia: a randomized, open-label, controlled trial. Int Clin Psychopharmacol. 2012; 27(5):267-274.

11. Morosini PL, Magliano L, Brambilla L, Ugolini S, Pioli R. Development, reliability and acceptability of a new version of the DSM-IV social and occupational functioning assessment scale (SOFAS) to assess routine social functioning. Acta Psychiatr Scand. 2000;101(4): 323-329.

12. Kern RS, Nuechterlein KH, Green MF, et al. The MATRICS consensus cognitive battery, part 2: co-norming and standardization. Am J Psychiatry. 2008;165(2):214-220.

13. Shi C, Kang L, Yao S, et al. The MATRICS consensus cognitive battery (MCCB): Co-norming and standardization in China. Schizophr Res. 2015; 169(1-3):109-115.

14. Kay SR, Fiszbein A, Opler LA. The positive and negative syndrome scale (PANSS) for schizophrenia. Schizophr Bull. 1987;13(2): 261-276.

15. Guy W. Clinical global impressions (CGI) scale. In: Rush A, editor. Handbook of Psychiatric Measures. American Psychiatric Association, Washington: DC; 2000:100-102.

16. Munetz MR, Benjamin S. How to examine patients using the abnormal involuntary movement scale. Hosp Community Psychiatry. 1988; 39(11):1172-1177.

17. Barnes TR. A rating scale for drug-induced akathisia. Br J Psychiatry. 1989;154:672-676.

18. Simpson GM, Angus JW. A rating scale for extrapyramidal side effects. Acta Psychiatr Scand Suppl. 1970;212:11-19.

19. Schreiner A, Lahaye M, Peuskens J, et al. Paliperidone extended-release in patients with non-acute schizophrenia previously unsuccessfully treated with other oral antipsychotics. Expert Opin Pharmacother. 2014; 15(5):593-603.

20. Locklear J, Lasser R, Rodriguez S, Turkoz I, Feifel D. Maintenance Therapy with Long-Acting Risperidone: Functioning and Quality of Life in Patients with Schizophrenia or Schizoaffective Disorder. Presented at the International Congress on Schizophrenia Research: 2005 April 2-7, Savannah, GA.

21. Mausbach BT, Moore R, Bowie C, Cardenas V, Patterson TL. A review of instruments for measuring functional recovery in those diagnosed with psychosis. Schizophr Bull. 2009;35(2):307-318.

22. Tianmei S, Liang S, Yun'ai S, et al. The Chinese version of the personal and social performance scale (PSP): validity and reliability. Psychiatry Res. 2011;185(1-2):275-279.

23. Corrigan PW, Watson AC. Understanding the impact of stigma on people with mental illness. World Psychiatry. 2002;1(1):16-20. 
24. Barnes TR, Leeson VC, Mutsatsa SH, Watt HC, Hutton SB, Joyce EM. Duration of untreated psychosis and social function: 1-year follow-up study of first-episode schizophrenia. Br J Psychiatry. 2008;193(3): 203-209.

25. Shi C, He Y, Cheung EF, Yu X, Chan RC. An ecologically valid performance-based social functioning assessment battery for schizophrenia. Psychiatry Res. 2013;210(3):787-793.

26. Kiejna A, Piotrowski P, Misiak B, et al. Predictors of vocational status in schizophrenia patients - Results from the Polish nationwide survey. Int J Soc Psychiatry. 2015;61(8):824-831.

27. Strassnig MT, Raykov T, O'Gorman C, et al. Determinants of different aspects of everyday outcome in schizophrenia: the roles of negative symptoms, cognition, and functional capacity. Schizophr Res. 2015;165(1): 76-82.

28. Addington J, Addington D. Neurocognitive and social functioning in schizophrenia: a 2.5 year follow-up study. Schizophr Res. 2000; 44(1):47-56.
29. Keefe RS, Silva SG, Perkins DO, Lieberman JA. The effects of atypical antipsychotic drugs on neurocognitive impairment in schizophrenia: a review and meta-analysis. Schizophr Bull. 1999;25(2):201-222.

30. Mishara AL, Goldberg TE. A meta-analysis and critical review of the effects of conventional neuroleptic treatment on cognition in schizophrenia: opening a closed book. Biol Psychiatry. 2004;55(10):1013-1022.

31. Keefe RS, Sweeney JA, Gu H, et al. Effects of olanzapine, quetiapine, and risperidone on neurocognitive function in early psychosis: a randomized, double-blind 52-week comparison. Am J Psychiatry. 2007;164(7):1061-1071.

32. Molina V, Taboada D, Aragüés M, et al. Greater clinical and cognitive improvement with clozapine and risperidone associated with a thinner cortex at baseline in first-episode schizophrenia. Schizophr Res. 2014; 158(1-3):223-229.

33. Kolotkin RL, Corey-Lisle PK, Crosby RD, et al. Impact of obesity on health-related quality of life in schizophrenia and bipolar disorder. Obesity (Silver Spring). 2008;16(4):749-754.

\section{Publish your work in this journal}

Neuropsychiatric Disease and Treatment is an international, peerreviewed journal of clinical therapeutics and pharmacology focusing on concise rapid reporting of clinical or pre-clinical studies on a range of neuropsychiatric and neurological disorders. This journal is indexed on PubMed Central, the 'PsycINFO' database and CAS, and is the official journal of The International Neuropsychiatric Association (INA). The manuscript management system is completely online and includes a very quick and fair peer-review system, which is all easy to use. Visit http://www.dovepress.com/testimonials.php to read real quotes from published authors. 